\section{Remission After Radiotherapy for a Patient With Chemotherapy-Refractory HIV- Associated Primary Effusion Lymphoma}

A 51-year-old HIV-infected man presented with a 4-week history of shortness of breath, right-sided chest pain, drenching night sweats, and 5-kg weight loss. He had been diagnosed with HIV infection 23 years previously. Investigations showed anemia (hemoglobin, $9.8 \mathrm{~g} / \mathrm{d}$ ), an elevated C-reactive protein of 138.6 $\mathrm{mg} / \mathrm{L}$, a CD4 count of 550 cells/ $\mu \mathrm{L}$, HIV viral load of 6800 copies/ $\mathrm{mL}$, and plasma Kaposi sarcoma-associated herpes virus (KSHV)/ human herpes virus 8 (HHV8) DNA of 70,000 copies/mL. A chest radiograph showed a moderately large right-sided pleural effusion. He was treated empirically for atypical pneumonia. Despite a course of antibiotics, the patient continued to complain of breathlessness. A chest radiograph 10 weeks later revealed a right-sided loculated effusion and pleural thickening. A computed tomography (CT) scan showed multiple pleural masses, the largest measuring $6.8 \times 4.3 \mathrm{~cm}$, located at the right posterolateral base, a pericardial nodule adjacent to the right atrium, a right-sided pleural effusion, and multiple small volume supraclavicular, axillary, retroperitoneal, pelvic, and inguinal adenopathy. Biopsy of the right base pleural mass identified a neoplastic infiltrate composed of large lymphoid cells with irregular pleomorphic nuclei. Many of the cells had eccentrically placed nuclei and abundant cytoplasm, giving the tumor a plasmablastic appearance (Fig 1A). Immunohistochemistry demonstrated that the tumor cells were positive for CD138 (Fig 1B), epithelial membrane antigen, and HHV8 (Fig 1C) and showed lambda light chain restriction. Epstein-Barr virus in situ hybridization was negative, and mindbomb homolog 1 showed a proliferation fraction of over $90 \%$. The B-cell markers CD20 (Fig 1D), CD79a, and paired box gene 5 were negative. The radiologic, histologic, and serologic findings were those of HIVassociated primary effusion lymphoma (PEL). He was commenced on a regimen of cyclophosphamide, doxorubicin, vincristine, and prednisolone (CHOP) chemotherapy with prophylactic intrathecal methotrexate and simultaneously started on antiretroviral therapy (ART; tenofovir, emtricitabine, and efavirenz). A CT scan after six cycles of CHOP chemotherapy showed significant disease response, with the right posterior pleural mass reduced to $4.8 \times 3.2$ $\mathrm{cm}$ and complete resolution of the pleural effusion. However, a positron emission tomography/CT scan assessment after eight cycles of CHOP chemotherapy showed an enlarging $\left[{ }^{18} \mathrm{~F}\right]$ fluorodeoxyglucose-avid pleural mass $(5 \times 5 \mathrm{~cm})$. Biopsy of the posterior pleural mass showed histological appearances identical to previous biopsy, validating a diagnosis of refractory PEL. The patient started

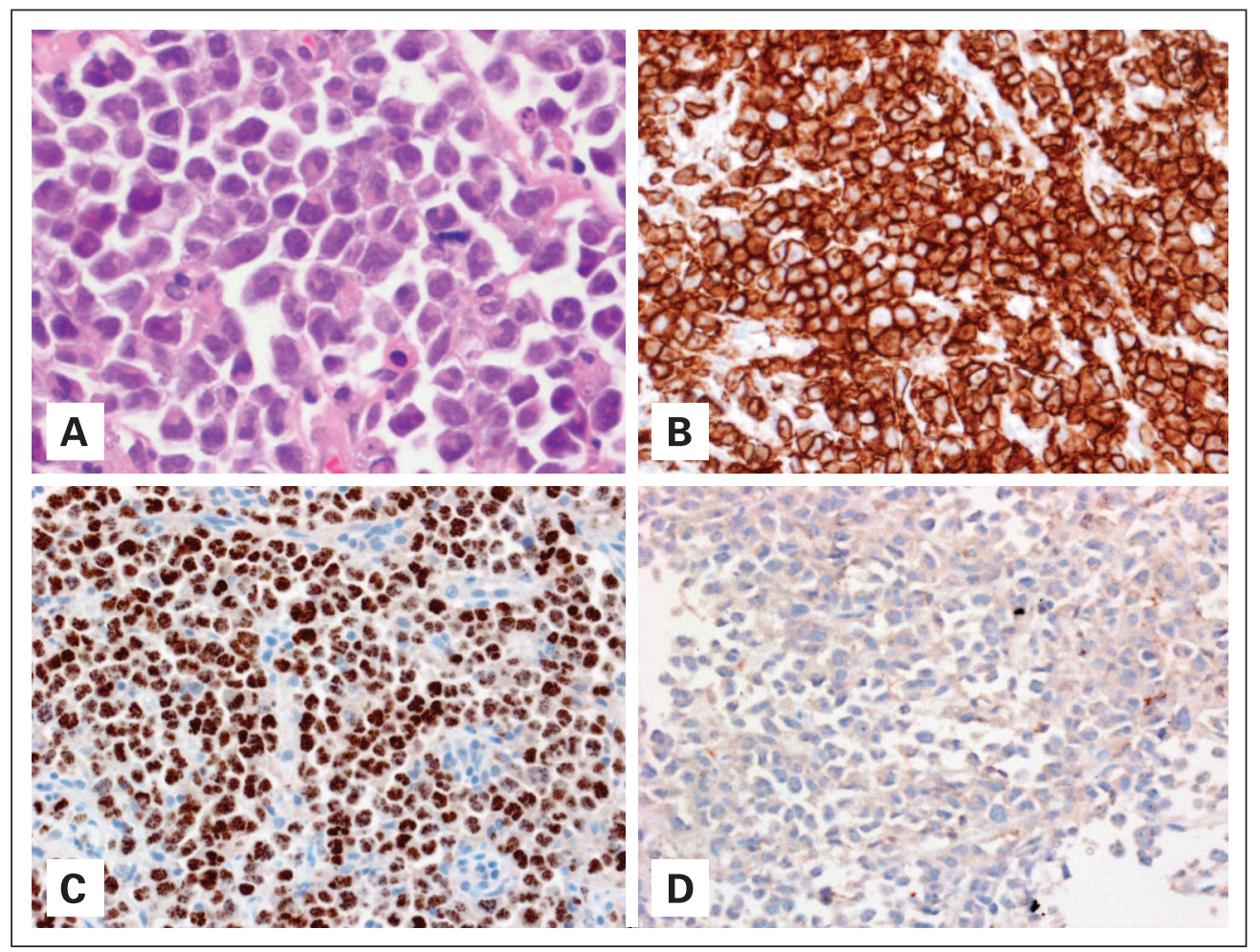

Fig 1. 

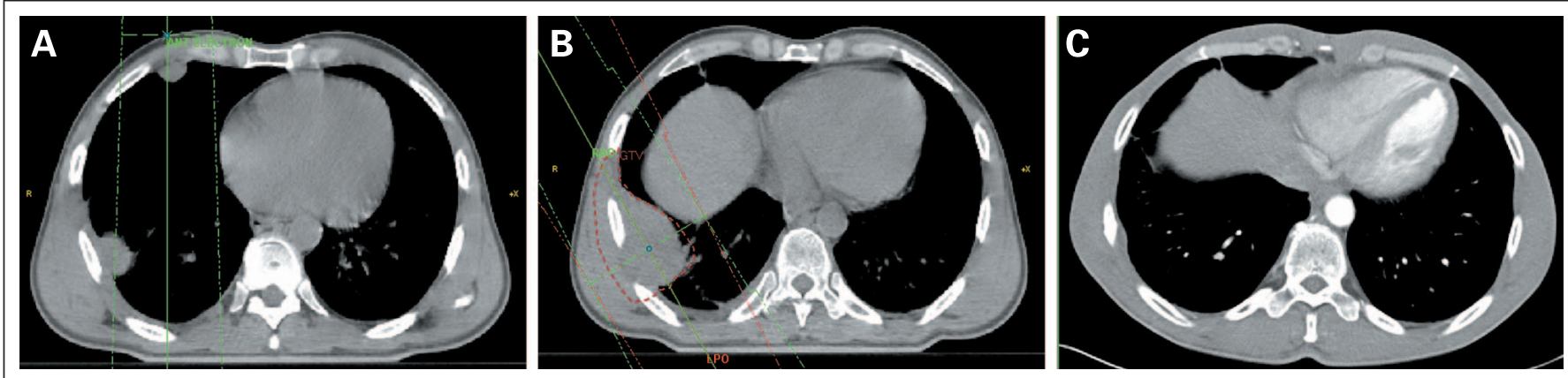

Fig 2.

second-line chemotherapy with etoposide, methylprednisolone, cytarabine, and cisplatin (ESHAP) with a view to consolidating treatment with high-dose chemotherapy and peripheral blood stem cells transplantation if significant disease response was achieved. Unfortunately, CT scan assessment after two cycles of ESHAP chemotherapy showed significant disease progression, with the right posterior pleural base mass now measuring $7 \times 6 \mathrm{~cm}$. He was commenced on third-line combination chemotherapy with ifosphamide, carboplatin, and etoposide (ICE), but despite three cycles of ICE chemotherapy, the right-sided posterolateral chest mass continued to increase in size, measuring $11.5 \times 7.0 \mathrm{~cm}$, and also an enlarging anterior pleural-based mass increased, now measuring $1.6 \times 2.1 \mathrm{~cm}$. Plasma KSHV remained detectable at 20,000 copies $/ \mathrm{mL}$. At this stage, further chemotherapy treatment was judged to be futile. The two masses received radiotherapy as follows: Both had fields placed at CT-based virtual simulation. A direct $12 \mathrm{MeV}$ electron field was used for the small pleurally based anterior mass (Fig 2A), and oblique tangential $6 \mathrm{MV}$ photon field used for the larger inferior posterolateral mass (Fig 2B). Both received $20 \mathrm{~Gy}$ in 5 fractions; the former prescribed to the $90 \%$ isodose, the latter to the mid point. A CT scan performed 4 months after radiotherapy showed complete resolution of the large pleural base mass (Fig 2C) and also of the anterior pleural mass. Twelve months after completing radiotherapy, the patient remained well and symptom free, and had a plasma KSHV DNA below the limit of quantification $(<100$ copies $/ \mathrm{mL})$. Overall survival since original histological diagnosis is now 23 months.

PEL is a rare HIV-associated malignancy representing around $3 \%$ to $4 \%$ of all HIV-related non-Hodgkin's lymphoma (NHL) with poor clinical outcome. ${ }^{1,2,3}$ PEL is defined by serous body cavity effusions and expression of KSHV, also known as HHV8, in their tumor cells. ${ }^{1-5}$ Over $70 \%$ of cases display concurrent EpsteinBarr virus infection. The optimal treatment for PEL has not yet been defined. In most cases, patients are treated with $\mathrm{CHOP}$ chemotherapy together with introduction of highly active ART. ${ }^{1}$ Despite this, durable responses are rarely seen and survival is short with median survival averaging 3 to 6 months. ${ }^{1,4,6,7}$ To our knowledge, this is the first description of a patient with HIV-associated PEL in who radiation treatment was associated with prolonged clinical, radiological, and serological response after failing three regimens of systemic chemotherapy treatments. Given the chemotherapyrefractory behavior of the disease, the dramatic response to radiotherapy was unexpected and surprising. Our experience suggests
PEL is sensitive to radiation treatment and should be considered as part of the treatment recommendation for patients with chemotherapy-refractory PEL-associated solid masses. It would also be interesting to explore treating PEL patients with consolidation radiation therapy for any residual histologically proven masses following conventional CHOP chemotherapy or with total-body irradiation, whereas the role of high-dose chemotherapy and peripheral stem-cell transplantation in refractory or relapsed HIVassociated PEL is yet to be ascertained. ${ }^{8}$

\section{Anna Cassoni, Usman Ali, and Judith Cave \\ Department of Oncology, University College London Hospital, London, United Kingdom}

\section{Simon G. Edwards}

Department of Genitourinary Medicine, Mortimer Market Centre, Camden Primary Care Trust, London, United Kingdom

\section{Alan Ramsay}

Department of Pathology, University College London Hospital, London, United Kingdom

\section{Robert F. Miller}

Centre for Sexual Health and HIV Research, Research Department of Infection and Population Health, University College London, London, United Kingdom

\section{Siow Ming Lee}

Department of Oncology, University College London Hospital; University College London Cancer Institute, University College London, London, United Kingdom

\section{ACKNOWLEDGMENT}

Supported by University College London Hospital/University College London Comprehensive Biomedical Research Centre and Gerard Kingdon for secretarial assistance.

\section{AUTHORS' DISCLOSURES OF POTENTIAL CONFLICTS OF INTEREST}

The author(s) indicated no potential conflicts of interest.

\section{REFERENCES}

1. Simonelli $C$, Spina $M$, Cinelli $R$, et al: Clinical features and outcome of primary effusion lymphoma in HIV-infected patients: A single-institution study J Clin Oncol 21:3948-3954, 2003

2. Knowles DM: Etiology and pathogenesis of AIDS-related non-Hodgkin's Iymphoma. Hematol Oncol Clin North Am 17:785-820, 2003

3. Carbone A, Gaidano G: HHV-8 positive body cavity-based lymphoma: A novel lymphoma entity. British J Haematol 97:515-522, 1997

4. Cesarman E, Chang Y, Moore PS, et al: Kaposi's sarcoma-associated herpes virus-like DNA sequences in AIDS-related body cavity-based lymphomas. N Engl J Med 332:1186-1191, 1995 
5. Nador RG, Cesarman E, Chadburn A, et al: Primary effusion lymphoma: A distinct clinicopathologic entity associated with the Kaposi's sarcoma-associated herpes virus. Blood 88:645-656, 1996

6. Ansari MQ, Dawson DB, Nador R, et al: Primary body cavity-based AIDSrelated lymphomas. Am J Clin Pathol 105:221-229, 1996

7. Boulanger E, Gérard L, Gabarre J, et al: Prognostic factors and outcome of human herpes virus 8-associated primary effusion lymphoma in patients with AIDS. J Clin Oncol 23:4372-4380, 2005
8. Waddington TW, Aboulafia DM: Failure to eradicate AIDS-associated primary effusion lymphoma with high-dose chemotherapy and autologous stem cell reinfusion: Case report and literature review. AIDS Patients and STDs 18:67-73, 2004

DOI: 10.1200/JCO.2008.18.3350; published online ahead of print at www.jco.org on October 13, 2008

\section{Chronic Lymphocytic Leukemia With Eyelid Involvement Responding to Alemtuzumab}

A 61-year-old man was diagnosed in another hematological department as having chronic lymphocytic leukemia (CLL) in 1998. One year later, because of progressive stage II CLL characterized by doubling lymphocytes count after 6 months, increase of splenomegaly, and the appearance of B symptoms, the patient was treated with fludarabine, obtaining partial remission of disease. Starting from 2000, due to progression of disease, the patient underwent a cyclophosphamide, doxorubicin, vincristine, and prednisolone regimen plus interferon- $\alpha$ as maintenance therapy for 2 years; thereafter the successive relapse was treated with chlorambucil plus metilprednisolone in 2003 and chlorambucil in 2005, respectively. In January 2006, he presented lymphoadenopaties between 1.5 and $2.5 \mathrm{~cm}$ in his right armpit, and inguinal bilaterally inferior margin of spleen $2 \mathrm{~cm}$ after the costs margin, absent of B symptoms. A total-body computed tomography scan confirmed multiple lympoadenomegalies in the mediastinum, abdomen, and splenomegaly. An emocromocytomet- ric analysis presented a WBC of $27,280 / \mathrm{mm}^{3}$ (neutrophil, 2,000/ $\mathrm{mm}^{3}$; leukocyte, $23,000 / \mathrm{mm}^{3}$ ); hemoglobin of $14.3 \mathrm{~g} / \mathrm{dL}$; medium cellular volume of $84.6 \mathrm{fl}$; and platelets of $204 \times 10^{9} / \mathrm{L}$. A peripheral blood smear showed more than $50 \%$ of big lymphocytes (its size was double in respect to erythrocyte size); for these reasons we classified this form as atypical chronic lymphocytic leukemia (mixed CLL). Bone marrow aspiration showed $55 \%$ of infiltration by mature lymphocytes, and a bone marrow biopsy confirmed the same diffuse infiltration. Cytofluorimetric analysis of peripheral blood showed positivity for CD5, $\mathrm{CD} 5 / \mathrm{CD} 19, \mathrm{CD} 23$, and $\kappa$; at the same time the positivity of CD38 (73\%) and ZAP-70 (41\%) was detected. Fluorescent in situ hybridization analysis showed trisomy of chromosome 12, as expected by morphological analysis and $17 \mathrm{p}$ deletion. The mutational status of variable region of heavy chain immunoglobin discovered a mutated status (variable heavy chain 3-49). Because of stable stage II disease with bad biologic parameter in theheavily pretreated patient, a stringent follow-up was adopted. In October 2006, at routine check-up, the patient showed a bilateral painless nodular infiltration of the eyelids, similar to multiple chalazions. Two nodules were localized in each
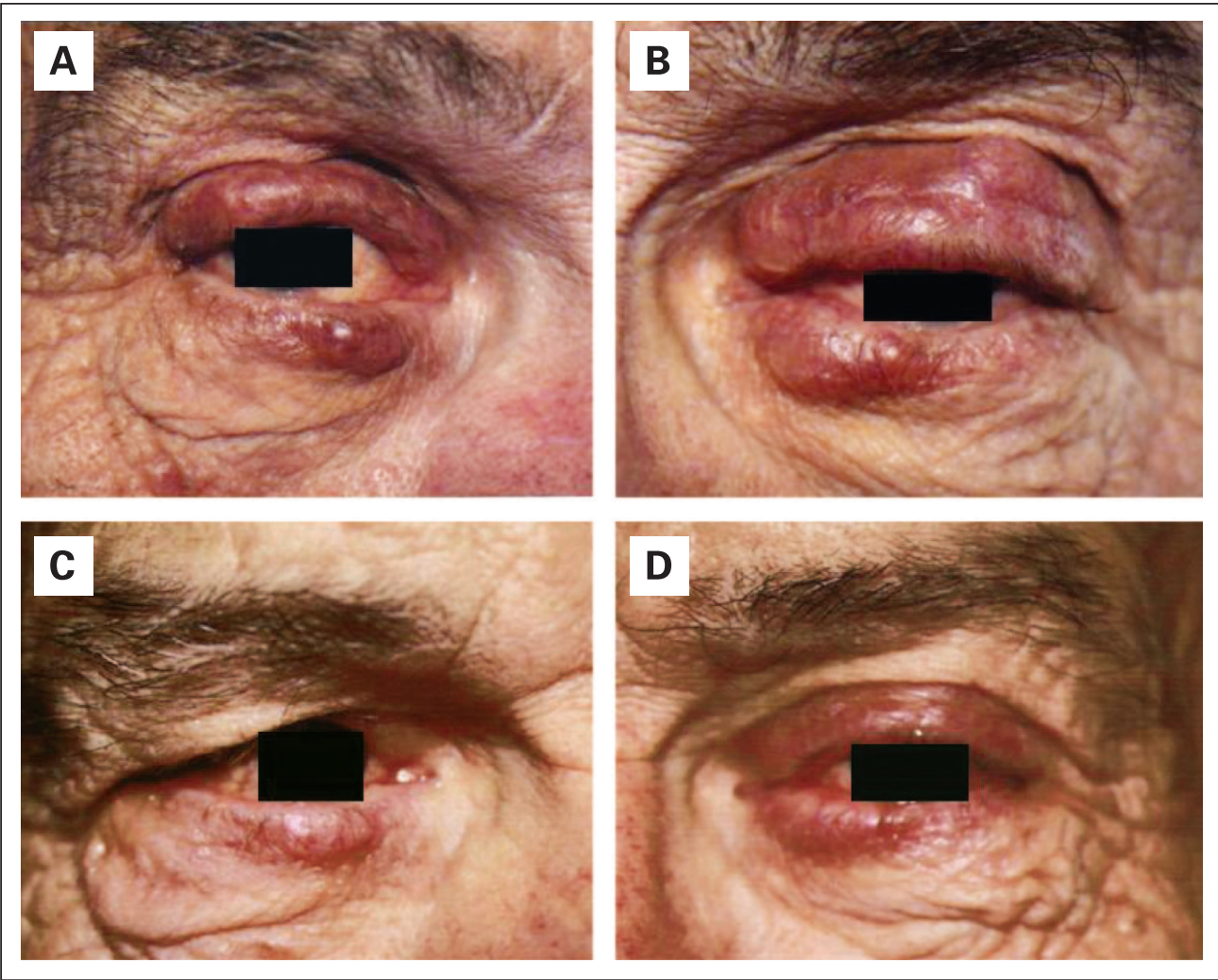

Fig 1. 